\title{
The lexical nature of alpha-beta oscillations in context-driven word production
}

\author{
Vitória Piai $^{\mathrm{a}, \mathrm{b}, *}$, Jana Klaus ${ }^{\mathrm{a}, \mathrm{c}}$, Elena Rossetto ${ }^{\mathrm{a}}$ \\ ${ }^{a}$ Radboud University, Donders Centre for Cognition, Nijmegen, the Netherlands \\ ${ }^{\mathrm{b}}$ Radboud University Medical Center, Donders Institute for Brain Cognition and Behaviour, Department of Medical Psychology, Nijmegen, the \\ Netherlands \\ ${ }^{\mathrm{c}}$ Max Planck Institute for Human Cognitive and Brain Sciences, Leipzig, Germany
}

\section{A R T I C L E I N F O}

\section{Keywords:}

Desynchronisation

Lexical access

Neuronal mechanism

Picture-word interference

Prediction

Semantic memory

\begin{abstract}
A B S T R A C T
In context-driven word production, picture naming is faster following constrained than neutral sentential contexts (e.g., "The farmer milked the... [picture]" vs. "The child drew a... [picture]", followed by the picture of a cow), suggesting conceptual-lexical pre-activation of the target response. Power decreases in the alpha-beta oscillatory band (8-25 Hz) are consistently found for constrained relative to neutral contexts prior to picture onset, when conceptual and lexical retrieval is ongoing. However, it remains a matter of debate whether the alpha-beta power decreases reflect (low-level) expectations of the visual input, conceptual and lexical retrieval, or motor preparation. The present study aimed at investigating the lexical-semantic nature of alphabeta oscillations. Participants performed context-driven picture naming with constrained and neutral contexts. In addition, an auditory distractor word was presented before picture onset. Distractors were either semantically related (e.g., "goat") or unrelated (e.g., "bean") to the picture to be named. Picture naming was faster with constrained than neutral contexts. Distractor type did not affect naming latencies nor the behavioural context effect. In the oscillatory brain responses, the context-related alpha-beta power decreases were observed throughout the prepicture interval when distractors were semantically unrelated to the picture, in line with previous findings. However, with semantically related distractors, the context effect was delayed until a period after distractor processing. Thus, alpha-beta power seems to be sensitive to the semantic relationship between the distractor word and the picture to be named. We interpret these results as suggesting that alpha-beta power decreases in context-driven word production reflect lexicalsemantic retrieval mechanisms.
\end{abstract}

\section{Introduction}

In recent years, studies employing scalp electrophysiology have started to shed light on the neurophysiological processes involved in word production (Aristei, Melinger, \& Abdel Rahman, 2011; Costa, Strijkers, Martin, \& Thierry, 2009; Piai, Roelofs, Jensen, Schoffelen, \& Bonnefond, 2014; Riès, Janssen, Burle, \& Alario, 2013; see for reviews; Ganushchak, Christoffels, \& Schiller, 2011; Indefrey, 2011; Munding, Dubarry, \& Alario, 2016; Strijkers \& Costa, 2011). By providing a direct measure of net neuronal activity, the electrophysiological signal plays a key role in informing theories about the relationship between cognitive processes and neuronal mechanisms, also outside of the language domain (e.g., Cohen, 2014; Hanslmayr, Staudigl, \& Fellner, 2012; Llorens et al., 2016;

\footnotetext{
${ }^{*}$ Corresponding author. Montessorilaan 3 6525HR, Nijmegen, the Netherlands.

E-mail address: v.piai@donders.ru.nl (V. Piai).
} 
Miller, Lundqvist, \& Bastos, 2018; Schroeder \& Lakatos, 2009). In particular, brain oscillations are proposed to form a fundamental neuronal mechanism that supports sensory and cognitive functions (Arnal \& Giraud, 2012; Hanslmayr, Staresina, \& Bowman, 2016; Siegel, Donner, \& Engel, 2012; Tallon-Baudry \& Bertrand, 1999). Oscillations are thought to enable modulation of the excitability of neuronal populations and, thus, enable synaptic communication (Buzsáki \& Draguhn, 2004; Siegel et al., 2012). Interestingly, studies examining both event-related potentials, which are phase-locked to an event, and oscillatory responses have shown differences between the two measures in terms of time course, neuronal generators and, thus, function (Davidson \& Indefrey, 2007; Laaksonen, Kujala, Hultén, Liljeström, \& Salmelin, 2012; Piai, Roelofs, Jensen, et al., 2014; Piai, Roelofs, \& Maris, 2014). These findings indicate that the study of brain oscillations can provide valuable information regarding the neurophysiological basis of language production processes.

For speech production in particular, early work firmly established the involvement of alpha-beta oscillations in preparation and execution of oral movements (e.g., Crone, Miglioretti, Gordon, \& Lesser, 1998; Salmelin, Hámáaláinen, Kajola, \& Hari, 1995; Salmelin \& Sams, 2002; Salmelin, Schnitzler, Schmitz, \& Freund, 2000). However, producing language also involves the retrieval and selection of concepts and words to be expressed. How these memory aspects of speaking are characterised in neuronal oscillations has been largely neglected in the literature (see for a review and discussion, Piai \& Zheng, 2019).

Language production is often studied with picture-naming paradigms. In this way, word production is initiated by a visual stimulus, the picture. However, in naturalistic situations, word production is typically embedded in a particular context (e.g., in the sentence of a speaker or her interlocutor). This situation can be approximated in an experimental setting by paradigms in which word production is guided by a sentence context (Griffin \& Bock, 1998). In such a paradigm, sentences can be manipulated in order to be semantically constrained or neutral towards the final word that has to be produced. This final word is omitted and instead a picture is presented for participants to name. Participants name the picture consistently faster if the context is constraining (Griffin \& Bock, 1998; Piai et al., 2016; Piai, Meyer, Dronkers, \& Knight, 2017; Piai, Roelofs, \& Maris, 2014; Piai, Roelofs, Rommers, \& Maris, 2015; Piai, Rommers, \& Knight, 2018), indicating that the initial stages of word production - presumably conceptual preparation and possibly also word retrieval - are initiated prior to the picture being presented (Piai et al., 2015). Thus, following constrained contexts, word planning can be initiated before a target to be named is presented (see also Shao \& Rommers, 2020 for additional findings). In the electrophysiological signal, this context effect is reflected in power decreases in the alpha-beta range (8-25 Hz) for constrained relative to neutral contexts already prior to picture onset (Piai et al., 2015, 2018, 2017; Piai, Roelofs, \& Maris, 2014). This suggests that conceptual and lexical retrieval in word production are supported by alpha-beta power decreases. However, in the literature, alpha-beta power decreases have been linked to attention and motor preparation (e.g., Brinkman, Stolk, Dijkerman, de Lange, \& Toni, 2014; Cheyne, 2013; Klimesch, 2012) besides lexical and semantic-memory processes (Fellner \& Hanslmayr, 2017; Mellem, Bastiaansen, Pilgrim, Medvedev, \& Friedman, 2012; Röhm, Klimesch, Haider, \& Doppelmayr, 2001; Weiss \& Mueller, 2012). Thus, it is not clear whether, in the current paradigm, the power decreases in the alpha-beta range are driven by attentional and motor preparation processes or can, in fact, be attributed to lexical processing as part of the utterance planning.

Two previous studies have used anatomical information to further constrain the interpretation of the alpha-beta power decreases in context-driven word production (Piai et al., 2015, 2018). Using source localisation, Piai et al. (2015) showed that the pre-picture power decreases were generated in the left temporal lobe, left inferior parietal lobe, and left inferior frontal gyrus, areas associated with stages of lexical access in word production (Indefrey \& Levelt, 2004) and with semantic memory (Binder, Desai, Graves, \& Conant, 2009). In a second study, patients with stroke-induced lesions to left temporal, inferior parietal, or inferior frontal cortex performed the context-driven picture naming task (Piai et al., 2018). Lesions comprising the left posterior temporal and inferior parietal lobes led to the attenuation of the context effect both in picture naming times and in the oscillations. These findings provided evidence for a causal link between the behavioural and oscillatory context effects, as well as for the involvement of the left posterior cortex in context-driven word production. Altogether, the results suggest that the alpha-beta power decreases likely reflect semantically constrained lexical-semantic retrieval (see for discussion Piai \& Zheng, 2019). This interpretation is in line with the hypothesis that alpha-beta power decreases are related to the richness of the information being encoded or retrieved as proposed by the "information via desychronisation hypothesis" (Hanslmayr et al., 2016, 2012).

In the present study, we aimed at further elucidating the lexical-semantic nature of alpha-beta power decreases in context-driven word production. Our participants read sentences that were either contextually constraining towards one final target word (e.g., "He poured wine in his") or neutral (e.g., "He saw something in his"). The target word completing the sentence (in this example "glass") was presented as a picture to be named. As mentioned above, following constrained contexts, conceptual and lexical activation can already begin in the absence of a picture to be named, i.e., during the pre-picture interval. To study the lexical-semantic nature of the pre-picture oscillatory effects, we presented auditory distractor words intervening during this pre-picture interval (i.e., between sentence offset and picture presentation). Distractor words were either semantically related (e.g., "mug") or unrelated (e.g., "bush") to the picture to be named, and thus (indirectly) also to the sentence, but never a valid response option (see Fig. 1A).

In this way, we made use of the rationale of the standard picture-word interference paradigm. In picture-word interference, participants are required to name a picture while ignoring a distractor word (Glaser \& Düngelhoff, 1984; Lupker, 1979). The relationship between the distractor and the picture can be manipulated to probe processing at different representational levels. For example, picture naming is slower if the distractor word is semantically related to the target word (e.g., picture "glass", distractor "mug") compared to an unrelated distractor (e.g., distractor "bush"). This semantic interference effect has been interpreted in terms of competition at the lexical level (e.g., Abdel Rahman \& Aristei, 2010; Piai, Roelofs, Jensen, et al., 2014; Roelofs, 1992). Thus, for our current design, in the constrained condition, a semantically related distractor competes for selection with the pre-activated target response during the pre-picture interval, whereas an unrelated distractor word can presumably be suppressed more easily because it does not present a valid response option. By contrast, in the neutral condition, the target response cannot be planned prior to picture 

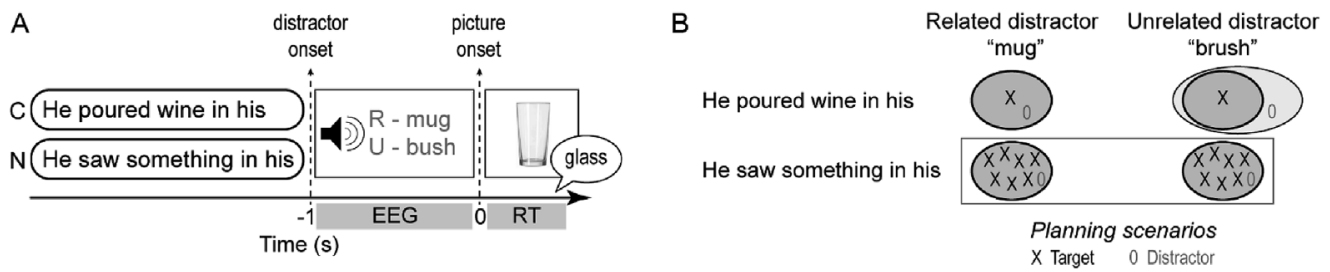

Fig. 1. A. Trial structure and example materials. $C=$ constrained sentence, $N=$ neutral sentence, $R=$ related distractor, $U=$ unrelated distractor. B. Schematic overview of the possible word planning scenarios. The target is indicated by a black X, the distractor is indicated by a grey 0 . For constrained contexts with related distractors, target and distractor pertain to the same set and are, therefore, stronger competitors, whereas unrelated distractors do not pertain to the same set as the target. Note how for neutral sentences, both planning scenarios are identical.

onset, so both the related and the unrelated distractors are just additional potential response options. These word-planning scenarios are illustrated in Fig. 1B.

It should be noted, however, that the current study employed a modification of the classical picture-word interference paradigm in that our pictures were presented in the context of a sentence. Additionally, our distractors were presented $1 \mathrm{~s}$ before picture onset, a stimulus onset asynchrony (SOA) at which no behavioural effect is expected (Damian \& Martin, 1999; Schriefers, Meyer, \& Levelt, 1990).

Regarding the brain oscillations, first of all, we expected to replicate the alpha-beta power decreases in the pre-picture interval as a function of sentence context (Piai et al., 2015, 2018, 2017; Piai, Roelofs, \& Maris, 2014). In addition, and most relevant for the present study, we also expected to observe differential modulation of the alpha-beta power decreases induced by the sentence context as a function of the semantic relatedness of the distractor to the picture. This prediction is derived from previous demonstrations that the pre-picture alpha-beta power decreases seem more related to conceptual and lexical processes rather than to motor or attentional processes (Piai et al., 2015, 2018). By contrast, if the alpha-beta power decreases reflect mainly motor preparation, then the semantic nature of the distractor word should not matter. Especially if the semantic nature of the distractor word differentially affects the oscillations, a stronger case can be made that alpha-beta oscillations also reflect lexical-semantic processes. However, given the lack of relevant theoretical and empirical literature, we could not derive a clear prediction about the specific direction of the power modulations, i.e., whether the presence of semantically related distractors would further decrease or rather increase the alpha-beta power decreases relative to the unrelated distractors.

\section{Materials and methods}

Materials and data of this study are available on the Open Science Framework (https://osf.io/fk6hq/).

\subsection{Participants}

Nineteen native speakers of Dutch (6 male, mean age $=22.6, S D=2.8$, range: $18-30$ ), voluntarily participated in the experiment for monetary compensation or course credits. The datasets of three participants ( 1 male) were not analysed due to excessive blinking resulting in the loss of a large number of trials ( $<70 \%$ of trials remaining). Thus, the complete dataset analysed and reported below contained data from 16 participants. All were right-handed, had normal or corrected-to-normal vision and no history of neurological or language deficits. Participants gave written consent after they were completely informed about the nature of the study. The experiment was approved by the Ethics Committee of Faculty of Social Sciences at Radboud University Nijmegen.

\subsection{Stimuli}

One-hundred coloured public-domain clipart images of common objects were taken from the internet and used as stimuli. For each picture, two sets of sentences were constructed for which the picture names were the last word of the sentences (target word). In one set, sentences were constructed such that the picture name was highly expected (constrained context, mean length: 7.6 words), whereas in the other set, no specific word was expected as the final word of the sentence (neutral context, mean length: 7.3 words).

To verify that the two context conditions differed with respect to the expected target word, we conducted an online experiment with 16 participants who did not take part in the main experiment. In this study, the sentences were presented up until the penultimate word of the sentence, thus missing the target noun, and the participants' task was to fill in the word which completed the sentence in the most meaningful way. The cloze probability for the target word was calculated as the proportion of participants who used the expected target picture name as completion. The cloze probability on the most common completion was calculated as the proportion of participants who used the same completion, different from the target word. This measure was used to check that the completion that we chose for the neutral sentences had a low plausibility. Lastly, the cloze probability on the second most common completion was calculated in order to check whether there was a strong effect of words that we did not consider as a completion for our sentences. Based on the pre-test, eight target words were replaced by a new target word with a higher cloze probability. The constrained sentences showed a mean cloze probability of 0.819 (median $=0.875, S D=0.149$ ). In the neutral condition, none of the sentences had a high-cloze probability for the target word (mean $=0.036$, median $=0, S D=0.092$ ). 
To create the distractor conditions, for each picture a word was chosen that was semantically related to the target word (e.g. "bush" for the target word "tree"). We note that, in the constrained condition, by extension, the semantically related distractor words are also related to the sentence, but never a valid response option. The unrelated condition was created by reassigning the distractors to different target words, ensuring that they shared no semantic relationship (e.g. "bush" for the target word "glass"). The distractors were chosen based on categorical relationships (e.g., for the related condition, an animal was chosen as a distractor if the picture was an animal, etc.). Unrelated items were created by reassigning the distractors to target words from a different category (e.g., an instrument as a distractor if the picture was an animal). The semantic association between distractors and pictures were confirmed by latent semantic analysis (LSA, Landauer, Foltz, \& Laham, 1998): mean (SD) LSA value for related distractors $=.152(0.168)$, for unrelated distractors $=.008(0.066), t(99)=7.85,95 \%$ CI $[0.108,0.180]$. Where possible, we matched distractor and target words for number of syllables and grammatical gender, and care was taken that there was no phonological onset overlap. For the constrained sentences, the related distractor words were rarely expected completions, as per responses provided by participants in the pre-test described above (i.e., in only $0.625 \%$ of the responses given by participants the distractor word we chose was used to complete the sentence). The auditory distractors were spoken by a female native speaker of Dutch and varied in duration from 0.301 to $0.866 \mathrm{~s}$, with an average length of $0.603 \mathrm{~s}(S D=0.132)$. The same related and unrelated distractors were used for the items in both the constrained and neutral condition, yielding 400 different combinations of sentences and distractor words.

\subsection{Design}

The experimental design included the within-participant variables sentence context (constrained vs. neutral) and distractor relatedness (semantically related vs. unrelated). The 400 picture-distractor combinations were split into four lists such that within one list, the same picture was shown in the constrained and the neutral context conditions. Thus, each picture was presented twice in each list. Whether a related or an unrelated distractor was presented with each picture and its context was counterbalanced (e.g., picture $\mathrm{X}$ in a neutral context was shown with a related distractor in list 1 and with an unrelated distractor in list 2; picture $\mathrm{X}$ in a constrained context was shown with an unrelated distractor in list 1 and with a related distractor in list 2). The sequence of experimental conditions per item across participants was counterbalanced using a sequentially balanced Latin square procedure. This method ensures that the order of the conditions per item is distributed evenly between participants, preventing confounds because all items appear in a fixed order with respect to their condition (Pollatsek \& Well, 1995). In the current study, this translates to four parallel lists, meaning that a particular item appeared in combinations AD, DA, BC, and CB for four different participants. This guaranteed that each participant saw each item in a constrained and unconstrained as well as a related and unrelated condition, but the combinations thereof, and the order in which the conditions were presented, differed across participants. The order of the 200 experimental trials per participant was pseudo-randomised using Mix (van Casteren \& Davis, 2006), with the constraints that the repetition of target or distractor words, respectively, was separated by at least 50 trials, and that the experimental conditions were not repeated for more than three consecutive trials.

\subsection{Procedure}

Participants were tested individually in an electrically and acoustically shielded cabin. The light and the volume of the speakers inside the cabin were kept the same across all participants, who were seated in front of a computer monitor and a microphone at a distance of approximately $60 \mathrm{~cm}$. Stimulus presentation and utterance recordings were controlled by Presentation software (Neurobehavioral Systems Inc., Berkeley, CA, www.neurobs.com). The sentences (presented word-by-word) and the pictures were presented on a white background at the centre of the screen. An experimental trial was structured as follows: After the presentation of a fixation cross for $0.5 \mathrm{~s}$, each word of the sentence was presented for $0.3 \mathrm{~s}$, interleaved with a blank screen presented for $0.2 \mathrm{~s}$. After the last word of the sentence disappeared, the auditory distractor word was presented. The picture was presented $1 \mathrm{~s}$ after the offset of the last word for a duration of $1 \mathrm{~s}$ (see Fig. 1), followed by a cue (***) for $2 \mathrm{~s}$ which indicated that participants were allowed to blink in this interval.

During the EEG preparation, participants were given a booklet containing all the experimental pictures and the corresponding target words, and were instructed to read it and try to use the respective labels during the experiment. Before the experiment, participants were instructed to fixate on the centre of the screen, to minimise head movement during the experimental blocks, and to blink only when a cue was presented on screen. Then, participants were given written instructions telling them that they had to read the sentence attentively and name the subsequent picture as quickly and accurately as possible. These instructions were practised in four trials. The practice round was repeated in case a participant did not follow the instructions. Next, a familiarisation phase was conducted with four trials in which the auditory distractor was introduced. Then, the experiment proper followed, consisting of 200 experimental trials divided into five blocks. Blocks were separated by short breaks. The whole session, including participant preparation, lasted approximately $90 \mathrm{~min}$.

\subsection{EEG data acquisition}

The EEG was recorded from 28 scalp electrodes mounted equidistantly in an elastic cap, positioned according to the international 10-20 system using the Acticap system and amplified with BrainAmps DC amplifiers (500 Hz sampling, 0.016-100 Hz band-pass). Each electrode was referenced online to the left mastoid and re-referenced offline to averaged mastoids. The electro-oculogram (EOG) was recorded horizontally from the electrodes placed on the left and right temples and vertically from the electrode positioned below 
the left eye. Electrode impedance was kept below $10 \mathrm{k} \Omega$ throughout the experiment.

\subsection{Statistical analysis of naming latencies}

The accuracy of the vocal responses was evaluated offline. Near synonyms (e.g., "flower" instead of "rose") were considered correct. Responses containing disfluencies, no utterances or wrong responses were coded as errors and the corresponding trials were excluded from the response time (RT) and EEG analyses (1.8\% of all trials).

RTs were measured manually using Praat (Boersma \& Weenink, 2013). Values larger than $1.5 \mathrm{~s}$ were removed (0.3\%). Statistical analyses were computed with linear mixed-effects models (LMEMs) using the lme4 package (version 1.1-13, Bates, Mächler, Bolker, \& Walker, 2015) in R (version 3.4.1, www.r-project.org). The factors context (neutral vs. constrained) and distractor relatedness (semantically related vs. unrelated) were sum-coded and included as fixed effects in the models. We used a maximal random-effects structure that included random intercepts and random slopes (for all fixed effects and their interactions) for both participants and items (Barr, Levy, Scheepers, \& Tily, 2013). Fixed effects were considered significant if their absolute $t$ value exceeded the value of 2 (Baayen, Davidson, \& Bates, 2008).

\subsection{EEG pre-processing}

The analyses were performed using FieldTrip version 20180131 (Oostenveld, Fries, Maris, \& Schoffelen, 2011). All trials excluded from the behavioural analysis were also excluded from the EEG analysis. Trials were cut from the raw data time-locked to picture presentation in segments of $1.2 \mathrm{~s}$ pre-picture onset to $0.3 \mathrm{~s}$ post-picture onset. Then, the raw signal in these segments was detrended (i.e., the mean of the whole epoch was subtracted from each sample point in the epoch, which is equivalent to baseline correction). This procedure follows previous investigations using the same paradigm (Piai et al., 2015, 2018, 2017; Piai, Roelofs, \& Maris, 2014). We then applied a low-pass zero-phase shift Butterworth filter with a cutoff frequency of $45 \mathrm{~Hz}$. For two participants, one channel was removed from the data due to excessive noise and subsequently interpolated using a spherical spline method (Perrin, Pernier, Bertrand, \& Echallier, 1989), as implemented in FieldTrip. For three participants with excessive blinking (but who still had more than $70 \%$ of valid data points), we used Independent Component Analysis to correct for eye movements (Jung et al., 2000, as implemented in FieldTrip). Between one and two components were removed from the data for these participants. For the remaining 13 participants, we visually inspected the data and rejected epochs with artefacts due to eye movements, blinks, muscle activity, or electrode drifting. In total, we rejected $6 \%$ of the data, equally distributed across conditions (per condition: constrained related $6 \%$, constrained unrelated 4\%, neutral related 7\%, neutral unrelated 5\%). After artefact rejection, an average of 39 out of 50 trials per participant remained in each condition.

\subsection{Time-resolved power analyses}

Time-resolved power was computed for the segments time-locked to picture onset at frequencies ranging between 1 and $40 \mathrm{~Hz}$, using a sliding time window of three cycles' length advanced in steps of $10 \mathrm{~ms}$ in the time dimension and of $1 \mathrm{~Hz}$ in the frequency dimension (see for a similar approach Piai, Roelofs, \& Maris, 2014). The data in each time window was multiplied with a Hanning taper and then the resulting signal was decomposed using the Fourier transform. Trials were then averaged per participant and per condition.

The sentence-distractor context effects (averaged over trials per participant) were statistically evaluated using non-parametric cluster-based permutation tests (Maris \& Oostenveld, 2007). The statistical tests included all available channels. Channels were set to have, on average, 4.4 neighbours. The frequency range $2-30 \mathrm{~Hz}$ was entered into the analysis to ensure we would capture potential power modulations due to the distractor word in frequency bands lower than $8 \mathrm{~Hz}$. In the time domain, the window between -0.85 and $0 \mathrm{~s}$ was included in the analysis. This selection was based on the fact that, right at auditory distractor onset, the signal is dominated by auditory evoked responses and contains little information about the lexical-semantic content of the distractor (see the Supplementary for results on phase consistency across trials, indicating strong evoked responses at auditory distractor onset). The time-frequency-channel space described above was scanned for adjacent time points, frequencies, and channels that showed similar differences across the conditions being compared. To calculate the permutation p-value, we used the Monte Carlo method with 1500 random permutations. A Monte Carlo cluster p-value below 5\% (two-tailed testing) was considered significant. With that, the false alarm rate used for determining statistical significance was controlled at the alpha level of 0.05 . The main effect of contextual constraint has been examined previously, showing robust and replicable alpha-beta power decreases in the pre-picture interval (Piai et al., 2017, 2018, 2015; Klaus, Schutter, \& Piai, 2019; Piai, Roelofs, \& Maris, 2014). Therefore, the focus of the present investigation was on the differences between the constrained and neutral conditions for each distractor type separately, to examine the differential contribution of distractor relatedness.

\section{Results}

\subsection{Distractor relatedness does not affect naming in context-driven word production}

Fig. 2 shows the mean naming latencies as a function of sentential constraint and distractor relatedness to the picture. In the constrained condition, mean naming latencies were $0.543 \mathrm{~s}(S D=0.187,95 \% \mathrm{CI}: 0.530,0.556)$ with related distractors and $0.546 \mathrm{~s}$ 


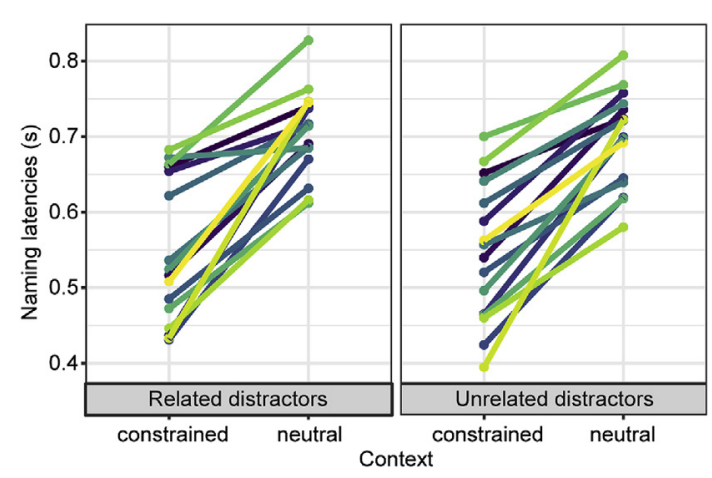

Fig. 2. Behavioral results. Individual mean naming latencies as a function of sentential constraint and distractor relatedness to the picture.

$(S D=0.187,95 \%$ CI: $0.533,0.559)$ with unrelated distractors. In the neutral condition, mean naming latencies were $0.700 \mathrm{~s}$ $(S D=0.152,95 \%$ CI: $0.689,0.711)$ with related distractors and 0.695 s $(S D=0.152,95 \%$ CI: $0.684,0.706)$ with unrelated distractors. Thus, naming latencies were consistently faster with constrained as opposed to neutral contexts (main effect of context: $\beta=-76.52, S E=8.44, t=-9.07, d=0.893$ ). However, naming latencies were not modulated by distractor relatedness $(\beta=0.77, S E=2.69, t=0.29, d=0.009)$. Also, there was no interaction of sentential constraint and distractor relatedness $(\beta=-1.82, S E=2.63, t=-0.69, d=0.022)$, suggesting that the context effect was comparable across distractor conditions.

\subsection{Distractor relatedness differentially modulates pre-picture alpha-beta band power}

Fig. 3 presents the relative power changes for the context effect (i.e., relative power changes for constrained versus neutral) for each distractor type separately.

For the related distractors, a cluster-based permutation test of the sentence-context effect revealed a statistically significant difference in power, with power decreases for the constrained relative to the neutral contexts (Monte Carlo $p=.046$ ). As shown in Fig. 3, the most prominent differences were found in the $5-20 \mathrm{~Hz}$ frequency range, in the $-0.350 \mathrm{~s}-0 \mathrm{~s}$ interval pre-picture presentation. The effect was mostly noted in posterior electrodes as shown in the topographical map on the right.

For the unrelated distractors, a cluster-based permutation test of the sentence-context effect also revealed a statistically significant difference in power, with power decreases for the constrained relative to the neutral contexts (Monte Carlo $p=.046$ ). As shown in Fig. 3, the most prominent differences were also found in the $5-20 \mathrm{~Hz}$ frequency range, but earlier relative to the effect for the related distractors, namely in the interval between $-0.650 \mathrm{~s}$ and $-0.170 \mathrm{~s}$ pre-picture presentation. The effect was mostly noted in left posterior electrodes, and stronger than for the related distractors as shown in the topographical map on the right. Electrodes pertaining to the cluster are shown in the configuration on top of the right panels. The topographical maps illustrate how the relative power decreases in the $5-20 \mathrm{~Hz}$ range are temporally distinct.

\subsubsection{Post-hoc analyses}

Additional, post-hoc analyses were run to further examine the impact of distractor relatedness on the context effect. An important
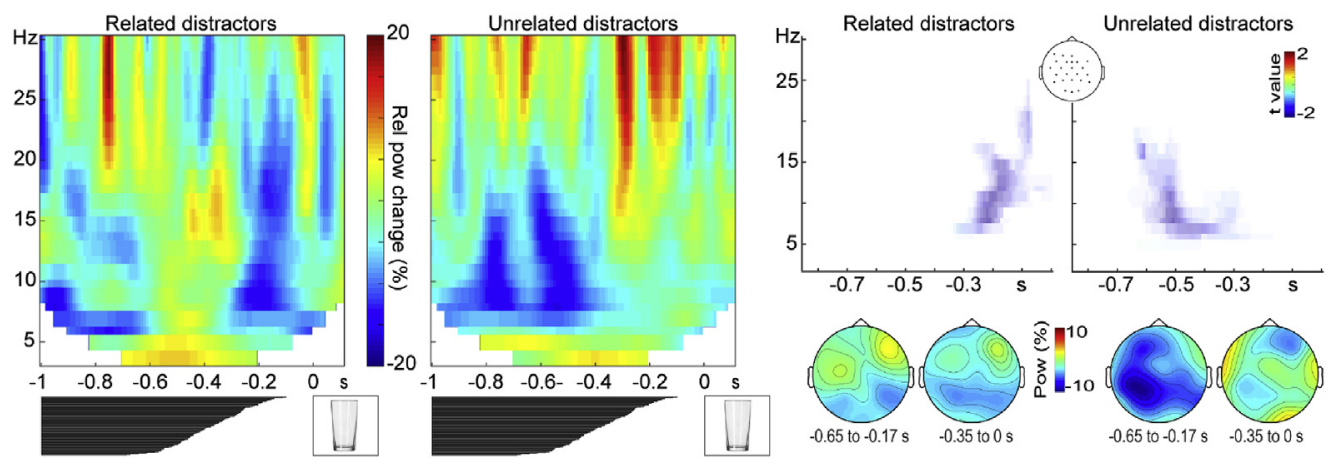

Fig. 3. Time-resolved power of the context effect (relative power changes for constrained versus neutral conditions) for related and unrelated distractors. Left. Relative power changes (constrained vs. neutral, normalised by their average) per distractor type, averaged over the channels shown in the right panel on the top. The images below the figure mark the events in the trial (i.e., distractor presentation and picture presentation). For the distractor, all the durations are plotted; the length of each bar indicates for how long the distractor was presented. Right. Statistical maps of the context effect (constrained versus neutral) per distractor type averaged over the channels pertaining to the significant cluster, shown on top. Only the data points participating in the significant clusters are shown. The topographical maps are shown for two intervals, indicated below each map. These intervals are derived from the results of the cluster-based permutation tests. 


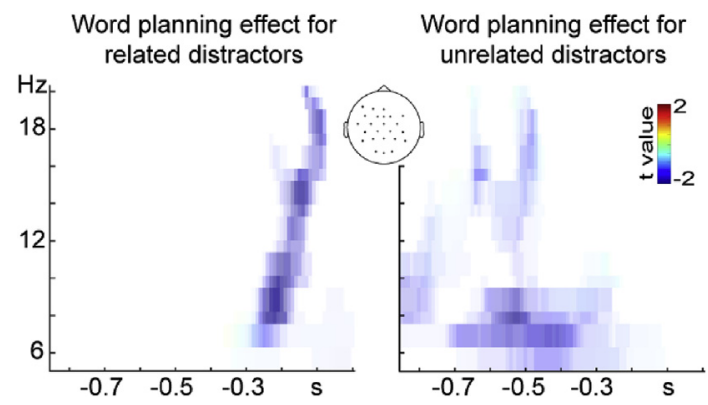

Fig. 4. Statistical maps of the word planning effects for each context-distractor relatedness versus the neutral condition (left: related distractors, right: unrelated distractors) averaged over the channels pertaining to the significant cluster, shown on top. Only the data points participating in the significant clusters are shown.

issue when analysing the oscillatory data from the pre-picture onset interval is that, unlike for the naming latency data, the design for the oscillatory data is not strictly speaking a 2 x 2 design. As illustrated in Fig. 1B, in the neutral condition, during distractor presentation in the pre-picture interval, both related and unrelated distractors are equally possible response options as they are equally strongly associated with the sentence context. Thus, with respect to word planning scenarios during the time window of interest (i.e., pre-picture), these two conditions are equivalent, which yields a design with one factor "word-planning context" with three levels (i.e., neutral contexts, constrained contexts with related distractors, and constrained contexts with unrelated distractors). Thus, to examine the planning context factor, we first averaged the two neutral conditions (i.e., related and unrelated distractors) for each participant (henceforth "neutral condition"). We then tested for the main effect of planning context using a cluster-based permutation F-test with the same parameters as described above, using all available channels, the frequency range of 2-30 Hz, and window between -0.850 and $0 \mathrm{~s}$. This revealed a statistically significant overall difference in power across the three conditions $(p=0.018)$. The most prominent differences were again found in the $5-20 \mathrm{~Hz}$ frequency range, between -0.800 and $0 \mathrm{~s}$. We followed up this main effect with pair-wise analyses in this time-frequency range (i.e., $5-20 \mathrm{~Hz}$, between -0.800 and $0 \mathrm{~s}$ ).

Fig. 4 presents the results of the inferential statistics for the two comparisons between the neutral condition and the specific context-relatedness conditions. For the unrelated distractors following a constrained context, a cluster-based permutation test revealed statistically significant power decreases relative to the neutral context (Monte Carlo $p=.008$ ). As shown in Fig. 4 , the most prominent differences were found in the $5-20 \mathrm{~Hz}$ frequency range for the interval between $-0.800 \mathrm{~s}$ and $-0.200 \mathrm{~s}$ pre-picture presentation. The effect was mostly noted in the same electrodes as identified in the main analyses shown in Fig. 3. For the related distractors following a constrained context, a cluster-based permutation test revealed statistically significant power decreases relative to the neutral context (Monte Carlo $p=.028$ ). As shown in Fig. 4, the most prominent differences were also found in the 5-20 Hz frequency range, but again later relative to the effect for the unrelated distractors. The results were very similar when the timefrequency range was not limited a-priori (i.e., the frequency range of $2-30 \mathrm{~Hz}$ and window between -0.850 and $0 \mathrm{~s}$ was tested). For the unrelated distractors, the power differences were present in the same time-frequency range $(p=0.038)$, whereas for related distractors the power decreases were not robust $(p=0.060)$. Altogether, these results strongly parallel the findings of the main pairwise analyses shown in Fig. 3, validating the robustness of the current findings.

Finally, to further examine the extent to which the differences between the two distractor conditions are not due to differences in the configuration of the neural sources, but rather mainly temporal in nature, we estimated spectral power for the $-0.650 \mathrm{~ms}$ to $-0.170 \mathrm{~ms}$ window, using a Hanning taper. The topographical maps of power in the $5-20 \mathrm{~Hz}$ are shown in Fig. 5 for each condition separately. The four scalp distributions look similar, with differences mainly in the strength of the spectral variations. This suggests similar neuronal generators of the alpha-beta oscillations varying in energy only.

Taken together, the findings indicate that the distractors' lexical-semantic content modulates the context-related power decreases in the $5-20 \mathrm{~Hz}$ (alpha-beta) range, during the period when conceptual, and possibly lexical, processes associated with word production are ongoing. In other words, following constrained contexts, which enable the pre-activation of lexical-semantic information and, thus, the initiation of word planning for production, the lexical-semantic information of a (competing) distractor word affects the alpha-beta power decreases.

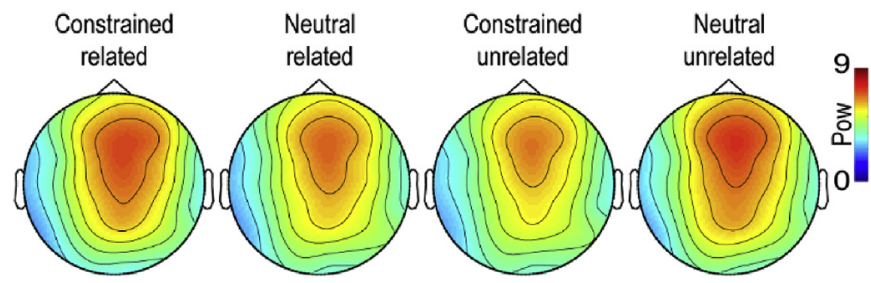

Fig. 5. Topographical maps of the time-averaged power spectra in the four conditions between 5 and $20 \mathrm{~Hz}$ for the $-0.650 \mathrm{~s}$ to $-0.170 \mathrm{~s}$ window. Pow $=$ power. 


\section{Discussion}

In the present study, we investigated whether neuronal oscillations in the alpha-beta frequency range are sensitive to lexicalsemantic information during context-driven word production. Participants read sentences that were either contextually constraining or neutral towards one target word, presented as a picture to be named. A distractor word, either semantically related or unrelated to the target picture, was presented before picture onset. We examined whether the lexical-semantic properties of the distractor word modulate the alpha-beta power oscillations associated with the sentence context effect.

As expected, the behavioral context effect was replicated (Griffin \& Bock, 1998; Piai, Roelofs, \& Maris, 2014). Naming latencies were, on average, $0.153 \mathrm{~s}$ faster with constrained than neutral contexts, confirming that sentence context affects the ease of word planning. The distractor manipulation did not show any effect on naming latencies. This is in line with the picture-word interference literature where no effects of semantically related distractors are found at long stimulus-onset asynchronies (Damian \& Martin, 1999; Schriefers et al., 1990). The lack of a behavioural semantic effect from distractor words with long pre-exposure is likely due to the fact that distractors can only interfere with certain word planning processes if these overlap in time and RTs reflect word planning processes with high fidelity. In the case of our paradigm, this situation is not as clear-cut. In the constrained condition, planning already starts before picture presentation, so the RTs will not reflect all word planning processes with high fidelity. Also, some early planning stages (e.g., conceptual retrieval) may already be partially completed by the time the distractor is presented, which diminishes the overlap between processing picture and distractor. Likewise, in the neutral condition, planning does not start until picture presentation, in which case the distractor word is presented too early to affect stages that will interact with the distractor's lexical-semantics (e.g., lexical selection).

With respect to the neuronal oscillations before picture presentation, our distractor manipulation modulated the oscillatory context effect in the alpha-beta range. Cluster-based permutation testing of the time-frequency representations indicated significant context effects (constrained vs. neutral) both for semantically related and unrelated distractors. In this interval, conceptual, and possibly even lexical, information is retrieved as a function of the sentence context (Jafarpour, Piai, Lin, \& Knight, 2017; Piai, Roelofs, \& Maris, 2014). However, the time interval during which these modulations occurred differed for the two types of distractor words. The alpha-beta context effect during a large portion of the pre-picture interval was replicated when distractors were semantically unrelated to the picture. Fig. 5 indicates that this effect is driven by less alpha-beta power in the constrained relative to the neutral context (the two topographical maps to the right in Fig. 5), replicating previous findings. By contrast, the alpha-beta context effect was absent for as long as semantically related distractors were processed concurrently with word planning stages for the picture to be named. In this case, alpha-beta power does not decrease for the constrained context (see Fig. 5, left most topographical map). Together, these findings indicate that the alpha-beta power decreases are sensitive to the lexical-semantic content of the distractor word. A cautionary note is in place regarding the task we used in that the sentence adds a dimensionality to the paradigm that may also influence the electrophysiological response to the auditory distractor.

Given that participants were familiarised with the pictures and their names prior to performing the task, it could be argued that the pictures were encoded during this familiarisation phase and episodic memories were retrieved during the task. However, the context-related behavioural and alpha-beta modulations have been consistently found across studies regardless of familiarisation (with familiarisation: Klaus et al., 2019; Piai, Roelofs, \& Maris, 2014; Piai et al., 2015, and the present findings; without familiarisation: Piai et al., 2016, 2017, 2018). Moreover, the modulations are found as a function of the language context, which is varied within pictures. All else being equal, episodic retrieval should be similar across the two context conditions because it involves the same pictures that were encoded during familiarisation.

We did not have a specific hypothesis about the direction of the power modulations for semantically related distractors. We can speculate about the direction of this effect based on the proposal that semantically related distractors interfere with word retrieval, as best illustrated in the picture-word interference paradigm, because they increase the competition amongst candidate representations (Roelofs, 1992). If we assume that semantically related distractors will influence conceptual and lexical retrieval processes ongoing during the pre-picture interval, competition is likely to occur. In the domain of episodic memory, alpha-beta power increases have been previously reported for retrieval under conditions of competing representations (Waldhauser, Johansson, \& Hanslmayr, 2012), which has been interpreted as indexing inhibition of the competing information. This previous finding allows us to speculate that alpha-beta power did not decrease in the constrained condition with semantically related distractors (see Fig. 5), as it did for unrelated distractors, because of the competition between the representations pre-activated by the sentence context and that of the distractor word.

Previous comprehension studies have also found that alpha and beta power decreases are sensitive to lexical-semantic information (Bastiaansen, van der Linden, Ter Keurs, Dijkstra, \& Hagoort, 2005; Mellem et al., 2012). For example, stronger alpha-beta power decreases (approximately between 8 and $21 \mathrm{~Hz}$ ) were observed roughly between 0.2 and $0.6 \mathrm{~s}$ after the presentation of open class words (i.e., nouns, verbs, and adjectives) relative to closed class words (i.e., determiners, prepositions, and conjunctions). Open class words carry more semantic information than do closed class words. Mellem et al. (2012) suggested that "alpha power decreases may be related to lexical-semantic retrieval operations" (page 4).

The "information via desychronisation hypothesis" (Hanslmayr et al., 2012) was proposed following the observation that alphabeta power decreases are repeatedly found in episodic memory tasks associated with successfully encoded memories (Khader \& Rösler, 2011; Klimesch, 1999; Klimesch, Doppelmayr, Schimke, \& Ripper, 1997). Hanslmayr and colleagues simulated firing rates of a neuronal population with varying degrees of synchronisation. By applying information theory to the degree of synchrony, they showed that neurones firing in synchrony convey less information, whereas desynchronised firing patterns (surfacing as power decreases) are better able to represent the richness of the information being encoded or retrieved. Although this hypothesis was put 
forward for the domain of episodic memory, it is plausible that certain neuronal principles from the episodic memory domain will also apply to the semantic memory domain and, by extension, to language production (e.g., Piai et al., 2016; see for discussion; Piai \& Zheng, 2019).

We would like to emphasise that the "information via desynchronisation hypothesis" was proposed for episodic memory and most of the evidence supporting this framework comes from the encoding rather than the retrieval stage (Hanslmayr et al., 2012). However, for language, retrieval from memory is likely the more relevant process, as it underlies both word comprehension and production. Given that alpha-beta power decreases are also found in comprehension (e.g., Bastiaansen et al., 2005; Mellem et al., 2012; Rommers, Dickson, Norton, Wlotko, \& Federmeier, 2017; Strauß, Kotz, Scharinger, \& Obleser, 2014), we propose that they may underlie a more fundamental computation of retrieving information from memory, be it for episodic memories, or for word comprehension and word production.

For the time being, our proposal that conceptual and lexical retrieval are enabled via power decreases of alpha-beta oscillations remains speculative. It is nevertheless at present the most parsimonious hypothesis of the neuronal mechanisms supporting lexicalsemantic retrieval with a direct link to the memory domain. Linking the domains of memory and language could be an important step for advancing our understanding of how (psycholinguistic) processes relate to neuronal mechanisms (Jafarpour et al., 2017; Piai et al., 2016). The memory domain has greatly benefited from insights provided by animal models, making mechanistic explanations at the neuronal level more likely to emerge (Buzsáki, 2005; Buzsáki \& Moser, 2013). Our knowledge about the neuronal mechanisms supporting language may be able to benefit from these mechanistic explanations.

In conclusion, we observed pre-picture alpha-beta power decreases for constrained relative to neutral contexts during the processing of semantically unrelated distractors. In addition, this oscillatory context effect was strongly attenuated during the processing of semantically related distractors. We interpret these results as evidence that alpha-beta desynchronisation carries lexical-semantic information. These findings and interpretation are in agreement with observations and theories from the episodic memory domain. Power modulations of alpha-beta oscillations may constitute a neuronal mechanism enabling not only encoding and retrieval of episodic memories, but also lexical-semantic retrieval in language production and comprehension.

\section{Funding}

This work was supported by the Netherlands Organisation for Scientific Research [grant numbers 451-17-003 to V.P. and Gravitation Grant 024.001.006 to the Language in Interaction Consortium]; and the German Research Foundation [grant numbers KL2933/2-1 and KL2933/3-1 to J.K.].

\section{CRediT authorship contribution statement}

Vitória Piai: Conceptualization, Formal analysis, Methodology, Project administration, Visualization, Writing - original draft, Writing - review \& editing. Jana Klaus: Conceptualization, Formal analysis, Methodology, Project administration, Visualization, Writing - original draft, Writing - review \& editing. Elena Rossetto: Investigation, Formal analysis, Project administration, Writing original draft.

\section{Appendix A. Supplementary data}

Supplementary data to this article can be found online at https://doi.org/10.1016/j.jneuroling.2020.100905.

\section{References}

Abdel Rahman, R., \& Aristei, S. (2010). Now you see it ... and now again: Semantic interference reflects lexical competition in speech production with and without articulation. Psychonomic Bulletin \& Review, 17(5), 657-661.

Aristei, S., Melinger, A., \& Abdel Rahman, R. (2011). Electrophysiological chronometry of semantic context effects in language production. Journal of Cognitive Neuroscience, 23, 1567-1586.

Arnal, L. H., \& Giraud, A.-L. (2012). Cortical oscillations and sensory predictions. Trends in Cognitive Sciences, 16(7), 390-398.

Baayen, R. H., Davidson, D. J., \& Bates, D. M. (2008). Mixed-effects modeling with crossed random effects for subjects and items. Journal of Memory and Language, 59(4), 390-412.

Barr, D. J., Levy, R., Scheepers, C., \& Tily, H. J. (2013). Random effects structure for confirmatory hypothesis testing : Keep it maximal. Journal of Memory and Language, 68(3), 255-278.

Bastiaansen, M. C. M., van der Linden, M., Ter Keurs, M., Dijkstra, T., \& Hagoort, P. (2005). Theta responses are involved in lexical-semantic retrieval during language processing. Journal of Cognitive Neuroscience, 17(3), 530-541.

Bates, D., Mächler, M., Bolker, B., \& Walker, S. (2015). Fitting linear mixed-effects models using lme4. Journal of Statistical Software, 67(1).

Binder, J. R., Desai, R. H., Graves, W. W., \& Conant, L. L. (2009). Where is the semantic system? A critical review and meta-analysis of 120 functional neuroimaging studies. Cerebral Cortex, 19(12), 2767-2796.

Boersma, P., \& Weenink, D. (2013). Praat: Doing phonetics by computer (Version 5.3.42). Retrieved from www.praat.org.

Brinkman, L., Stolk, A., Dijkerman, H. C., de Lange, F. P., \& Toni, I. (2014). Distinct roles for alpha- and beta-band oscillations during mental simulation of goaldirected actions. Journal of Neuroscience, 34(44), 14783-14792.

Buzsáki, G. (2005). Theta rhythm of navigation: Link between path integration and landmark navigation, episodic and semantic memory. Hippocampus, 15(7), $827-840$.

Buzsáki, G., \& Draguhn, A. (2004). Neuronal oscillations in cortical networks. Science, 304, 1926-1929.

Buzsáki, G., \& Moser, E. I. (2013). Memory, navigation and theta rhythm in the hippocampal-entorhinal system. Nature Neuroscience, $16(2), 130-138$.

van Casteren, M., \& Davis, M. H. (2006). Mix, a program for pseudorandomization. Behavior Research Methods, 38(4), 584-589.

Cheyne, D. O. (2013). MEG studies of sensorimotor rhythms: A review. Experimental Neurology, 245, 27-39. 
Cohen, M. X. (2014). A neural microcircuit for cognitive conflict detection and signaling. Trends in Neurosciences, 37(9), 480-490.

Costa, A., Strijkers, K., Martin, R. C., \& Thierry, G. (2009). The time course of word retrieval revealed by event-related brain potentials during overt speech. Proceedings of the National Academy of Sciences, 106(50), 21442.

Crone, N. E., Miglioretti, D. L., Gordon, B., et al. (1998). Functional mapping of human sensorimotor cortex with electrocorticographic spectral analysis. I. Alpha and beta event-related desynchronization. Brain, 121, 2271-2299.

Damian, M. F., \& Martin, R. C. (1999). Semantic and phonological codes interact in single word production. Journal of Experimental Psychology: Learning, Memory, and Cognition, 25(2), 345-361.

Davidson, D. J., \& Indefrey, P. (2007). An inverse relation between event-related and time-frequency violation responses in sentence processing. Brain Research, 1158, $81-92$.

Fellner, M.-C., \& Hanslmayr, S. (2017). Brain oscillations, semantic processing, and episodic memory. In M. Mody (Ed.). Neural mechanisms of language (pp. 63-80). Springer.

Ganushchak, L. Y., Christoffels, I. K., \& Schiller, N. O. (2011). The use of electroencephalography in language production research: A review. Frontiers in Psychology, 2(September), 208.

Glaser, W. R., \& Düngelhoff, F. J. (1984). The time course of picture-word interference. Journal of Experimental Psychology: Human Perception and Performance, 10(5), 640-654.

Griffin, Z. M., \& Bock, K. J. (1998). Constraint, word frequency, and the relationship between lexical processing levels in spoken word production. Journal of Memory and Language, 38, 313-338.

Hanslmayr, S., Staresina, B. P., \& Bowman, H. (2016). Oscillations and episodic memory: Addressing the synchronization/desynchronization conundrum. Trends in Neurosciences, 39(1), 16-25.

Hanslmayr, S., Staudigl, T., \& Fellner, M.-C. (2012). Oscillatory power decreases and long-term memory: The information via desynchronization hypothesis. Frontiers in Human Neuroscience, 6(April), 74.

Indefrey, P. (2011). The spatial and temporal signatures of word production components: A critical update. Frontiers in Psychology, 2(255), 1-16.

Indefrey, P., \& Levelt, W. J. M. (2004). The spatial and temporal signatures of word production components. Cognition, 92, $101-144$.

Jafarpour, A., Piai, V., Lin, J. J., \& Knight, R. T. (2017). Human hippocampal pre-activation predicts behavior. Scientific Reports, 7(1).

Jung, T. P., Makeig, S., Humphries, C., Lee, T. W. M.J.,M.Iragui, V., et al. . (2000). Removing electroencephalographic artifacts by blind source separation. Psychophysiology, 37, 163-178.

Khader, P. H., \& Rösler, F. (2011). EEG power changes reflect distinct mechanisms during long-term memory retrieval. Psychophysiology, 48(3), 362-369.

Klaus, J., Schutter, D. J. L. G., \& Piai, V. (2019). Transient perturbation of the left temporal cortex evokes plasticity-related reconfiguration of the lexical network. BioRxiv, 562827.

Klimesch, W. (1999). EEG alpha and theta oscillations reflect cognitive and memory performance: A review and analysis. Brain Research Reviews, 29(2-3), 169-195.

Klimesch, W. (2012). Alpha-band oscillations, attention, and controlled access to stored information. Trends in Cognitive Sciences, $16(12), 606-617$.

Klimesch, W., Doppelmayr, M., Schimke, H., \& Ripper, B. (1997). Theta synchronization and alpha desynchronization in a memory task. Psychophysiology, 34(2), 169-176.

Laaksonen, H., Kujala, J., Hultén, A., Liljeström, M., \& Salmelin, R. (2012). MEG evoked responses and rhythmic activity provide spatiotemporally complementary measures of neural activity in language production. NeuroImage, 60, 29-36.

Landauer, T. K., Foltz, P. W., \& Laham, D. (1998). An introduction to latent semantic analysis. Discourse Processes, 25(2-3), 259-284.

Llorens, A., Dubarry, A. S., Trébuchon, A., Chauvel, P., Alario, F.-X., \& Liégeois-Chauvel, C. (2016). Contextual modulation of hippocampal activity during picture naming. Brain and Language, 159, 92-101.

Lupker, S. J. (1979). Semantic nature of response competition in the picture-word interference task. Memory \& Cognition, 7, 485-495.

Maris, E., \& Oostenveld, R. (2007). Nonparametric statistical testing of EEG- and MEG-data. Journal of Neuroscience Methods, 164, 177-190.

Mellem, M. S., Bastiaansen, M. C. M., Pilgrim, L. K., Medvedev, A. V., \& Friedman, R. B. (2012). Word class and context affect alpha-band oscillatory dynamics in an older population. Frontiers in Psychology, 3, 97.

Miller, E. K., Lundqvist, M., \& Bastos, A. M. (2018). Working memory 2.0. Neuron, 100(2), 463-475.

Munding, D., Dubarry, A. S., \& Alario, F.-X. (2016). On the cortical dynamics of word production: A review of the MEG evidence. Language, Cognition and Neuroscience, 31(4), 441-462.

Oostenveld, R., Fries, P., Maris, E., \& Schoffelen, J.-M. (2011). FieldTrip: Open source software for advanced analysis of MEG, EEG, and invasive electrophysiological data. Computational Intelligence and Neuroscience, 1-9 2011.

Perrin, F., Pernier, J., Bertrand, O., \& Echallier, J. F. (1989). Spherical splines for scalp potential and current density mapping. Electroencephalography and Clinical Neurophysiology, 72(2), 184-187.

Piai, V., Anderson, K. L., Lin, J. J., Dewar, C., Parvizi, J., Dronkers, N. F., et al. (2016). Direct brain recordings reveal hippocampal rhythm underpinnings of language processing. Proceedings of the National Academy of Sciences, 113(40), 11366-11371.

Piai, V., Meyer, L., Dronkers, N. F., \& Knight, R. T. (2017). Neuroplasticity of language in left-hemisphere stroke: Evidence linking subsecond electrophysiology and structural connections. Human Brain Mapping, 38(6), 3151-3162.

Piai, V., Roelofs, A., Jensen, O., Schoffelen, J.-M., \& Bonnefond, M. (2014). Distinct patterns of brain activity characterise lexical activation and competition in spoken word production. PloS One, 9(2), e88674.

Piai, V., Roelofs, A., \& Maris, E. (2014). Oscillatory brain responses in spoken word production reflect lexical frequency and sentential constraint. Neuropsychologia, 53, 146-156.

Piai, V., Roelofs, A., Rommers, J., \& Maris, E. (2015). Beta oscillations reflect memory and motor aspects of spoken word production. Human Brain Mapping, 36(7), 2767-2780.

Piai, V., Rommers, J., \& Knight, R. T. (2018). Lesion evidence for a critical role of left posterior but not frontal areas in alpha-beta power decreases during contextdriven word production. European Journal of Neuroscience, 48(7), 2622-2629.

Piai, V., \& Zheng, X. (2019). Speaking waves: Neuronal oscillations in language production. Psychology of learning and motivation.

Pollatsek, A., \& Well, A. D. (1995). On the use of counterbalanced designs in cognitive research: A suggestion for a better and more powerful analysis. Journal of Experimental Psychology: Learning, Memory, and Cognition, 21(3), 785-794.

Riès, S. K., Janssen, N., Burle, B., \& Alario, F.-X. (2013). Response-locked brain dynamics of word production. PloS One, 8(3), e58197.

Roelofs, A. (1992). A spreading-activation theory of lemma retrieval in speaking. Cognition, 42, 107-142.

Röhm, D., Klimesch, W., Haider, H., \& Doppelmayr, M. (2001). The role of theta and alpha oscillations for language comprehension in the human electroencephalogram. Neuroscience Letters, 310(2-3), 137-140.

Rommers, J., Dickson, D. S., Norton, J. J. S., Wlotko, E. W., \& Federmeier, K. D. (2017). Alpha and theta band dynamics related to sentential constraint and word expectancy. Language, Cognition and Neuroscience, 32(5), 576-589.

Salmelin, R., Hámáaláinen, M., Kajola, M., \& Hari, R. (1995). Functional segregation of movement-related rhythmic activity in the human brain. NeuroImage, 2(4), 237-243.

Salmelin, R., \& Sams, M. (2002). Motor cortex involvement during verbal versus non-verbal lip and tongue movements. Human Brain Mapping, 16(2), 81-91.

Salmelin, R., Schnitzler, A., Schmitz, F., \& Freund, H. J. (2000). Single word reading in developmental stutterers and fluent speakers. Brain, 123, 1184-1202.

Schriefers, H., Meyer, A. S., \& Levelt, W. J. M. (1990). Exploring the time course of lexical access in language production: Picture-word interference studies. Journal of Memory and Language, 29, 86-102.

Schroeder, C. E., \& Lakatos, P. (2009). Low-frequency neuronal oscillations as instruments of sensory selection. Trends in Neurosciences, 32(1), 9-18.

Shao, Z., \& Rommers, J. (2020). How a question context aids word production: Evidence from the picture-word interference paradigm. Quarterly Journal of Experimental Psychology, 73(2), 165-173. https://doi.org/10.1177/1747021819882911. 
Siegel, M., Donner, T. H., \& Engel, A. K. (2012). Spectral fingerprints of large-scale neuronal interactions. Nature Reviews Neuroscience, 13(2), $121-134$.

Strauß, A., Kotz, S. a, Scharinger, M., \& Obleser, J. (2014). Alpha and theta brain oscillations index dissociable processes in spoken word recognition. NeuroImage, 97, $387-395$.

Strijkers, K., \& Costa, A. (2011). Riding the lexical speedway: A critical review on the time course of lexical selection in speech production. Frontiers in Psychology, 2 , 356.

Tallon-Baudry, C., \& Bertrand, O. (1999). Oscillatory gamma activity in humans and its role in object representation. Trends in Cognitive Sciences, 3(4), 151-162 (Retrieved from).

Waldhauser, G. T., Johansson, M., \& Hanslmayr, S. (2012). a/ß oscillations indicate inhibition of interfering visual memories. Journal of Neuroscience, 32(6), 1953-1961.

Weiss, S., \& Mueller, H. M. (2012). "Too many betas do not spoil the broth": The role of beta brain oscillations in language processing. Frontiers in Psychology, 3(June), 201. 